\title{
Dietary Total Antioxidants Capacity and Mortality in the PREDIMED study
}

Henríquez-Sánchez $\mathrm{P}^{1}$, Sánchez-Villegas $\mathrm{A}^{1}$, Ruano-Rodriguez $\mathrm{C}^{1}$, Gea $\mathrm{A}^{2}$, Lamuela-Raventós $\mathrm{RM}^{3}$, Estruch $\mathrm{R}^{4}$, Salas-Salvadó $\mathrm{J}^{5}$, Covas $\mathrm{I}^{6}$, Corella $\mathrm{D}^{7}$, Schröder $\mathrm{H}^{8}$, Gutierrez-Bedmar $\mathrm{M}^{9}$, Santos-Lozano $\mathrm{JM}^{10}$, Pintó $\mathrm{X}^{11}$, Arós $\mathrm{F}^{12}$, Fiol $\mathrm{M}^{13}$, Tresserra-Rimbau $\mathrm{A}^{3}$, Ros $\mathrm{E}^{14}$, Martínez-González $\mathrm{MA}^{2}$, Serra-Majem $\mathrm{Ll}^{1}$ on behalf of the PREDIMED Study Investigators

1. Centro de Investigación Biomédica en Red de Fisiopatología de la Obesidad y Nutrición, Instituto de Salud Carlos III, Madrid, Spain. Research Institute of Biomedical and Health Sciences, University of Las Palmas de Gran Canaria, Las Palmas de Gran Canaria, Spain.

2. Centro de Investigación Biomédica en Red de Fisiopatología de la Obesidad y Nutrición, Instituto de Salud Carlos III, Madrid, Spain. Department of Preventive Medicine and Public Health, University of Navarra, Pamplona, Navarra, Spain.

3. Centro de Investigación Biomédica en Red de Fisiopatología de la Obesidad y Nutrición, Instituto de Salud Carlos III, Madrid, Spain. Nutrition and Food Science Department, XaRTA, INSA, School of Pharmacy, University of Barcelona, Barcelona, Spain.

4. Centro de Investigación Biomédica en Red de Fisiopatología de la Obesidad y Nutrición, Instituto de Salud Carlos III, Madrid, Spain . Department of Internal Medicine, Institut d'Investigacions Biomèdiques August Pi i Sunyer (IDIBAPS), University of Barcelona, Barcelona, Spain.

5. Centro de Investigación Biomédica en Red de Fisiopatología de la Obesidad y Nutrición, Instituto de Salud Carlos III, Madrid, Spain. Human Nutrition Unit, School of Medicine, University Rovira i Virgili, Reus, Spain.

6. Centro de Investigación Biomédica en Red de Fisiopatología de la Obesidad y Nutrición, Instituto de Salud Carlos III, Madrid, Spain. Centro de Investigación Biomédica en Red de Fisiopatología de la Obesidad y Nutrición, Instituto de Salud Carlos II, Madrid, Spain.Cardiovascular Risk and Nutrition Research Unit, Institut Municipal d'Investigació Mèdica (IMIM), Barcelona, Spain.

7. Centro de Investigación Biomédica en Red de Fisiopatología de la Obesidad y Nutrición, Instituto de Salud Carlos III, Madrid, Spain. Department of Preventive Medicine and Public Health, University of Valencia, Valencia, Spain.

8. CIBER Epidemiología y Salud Pública (CIBERESP), Instituto de Salud Carlos III. Madrid.Spain. Cardiovascular Risk and Nutrition Research Group, Institut Municipal d'Investigació Medica (IMIM)Institut de Recerca del Hospital del Mar, Barcelona, Spain

9. Centro de Investigación Biomédica en Red de Fisiopatología de la Obesidad y Nutrición, Instituto de Salud Carlos III, Madrid, Spain. Department of Preventive Medicine, University of Malaga, Malaga, Spain

10. Centro de Investigación Biomédica en Red de Fisiopatología de la Obesidad y Nutrición, Instituto de Salud Carlos III, Madrid, Spain. Department of Family Medicine, Primary Care Division of Sevilla, San Pablo Health Center, Sevilla, Spain.

11. Centro de Investigación Biomédica en Red de Fisiopatología de la Obesidad y Nutrición, Instituto de Salud Carlos III, Madrid, Spain. Lipids and Vascular Risk Unit, Internal Medicine, Hospital Universitario de Bellvitge, Hospitalet de Llobregat, Barcelona, Spain

12. Centro de Investigación Biomédica en Red de Fisiopatología de la Obesidad y Nutrición, Instituto de Salud Carlos III, Madrid, Spain. Department of Cardiology, University Hospital of Alava, Vitoria, Spain 13. Centro de Investigación Biomédica en Red de Fisiopatología de la Obesidad y Nutrición, Instituto de Salud Carlos III, Madrid, Spain. Institute of Health Sciences, University of Balearic Islands, and Hospital Son Espases, Palma de Mallorca, Spain,

14. Centro de Investigación Biomédica en Red de Fisiopatología de la Obesidad y Nutrición, Instituto de Salud Carlos III, Madrid, Spain. Lipid Clinic, Endocrinology and Nutrition Service, Institut

d'Investigacions Biomèdiques August Pi Sunyer, Hospital Clinic, Barcelona, Spain

\section{Correspondence author:}

Dra. Patricia Henríquez Sánchez

e-mail: patrícia.henriquez@ulpgc.es

Tel. +34 928453402 Fax. +34 928453475 



\section{ABSTRACT}

Purpose: The aim of the present study was to assess the association between the dietary total antioxidant capacity, the dietary intake of different antioxidants and mortality in a Mediterranean population at high cardiovascular disease risk.

Methods: A total of 7,447 subjects from the PREDIMED study (multicenter, parallel-group, randomized controlled clinical trial), were analyzed treating data as an observational cohort. Different antioxidant vitamin intake and total dietary antioxidant capacity were calculated from a validated 137 item food frequency questionnaire at baseline and updated yearly. Deaths were ascertained through contact with families and general practitioners, review of medical records and consultation of the National Death Index. Cox regression models were fitted to assess the relationship between dietary total antioxidant capacity and mortality. Dietary total antioxidant capacity was estimated using FRAP assays (ferricreducing antioxidant power).

Results: A total of 319 deaths were recorded after a median follow-up of 4.3 years. Subjects belonging to the upper quintile of antioxidant capacity were younger, ex-smokers, with high educational level, more active and had higher alcohol intake. Multivariable-adjusted models revealed no statistically significant difference between total dietary antioxidant capacity and mortality (Q5 vs Q1 ref HR $=0.85$; 95\% CI 0.601.20) neither for the intake of all the vitamins studied.

Conclusions: No statistically significant association was found between antioxidant capacity and total mortality in elderly subjects at high cardiovascular risk.

Keywords: Dietary antioxidant capacity, antioxidants intake, mortality, PREDIMED 


\section{INTRODUCTION}

It has been widely reported that high consumption of fruits and vegetables might positively impact human health [1-4]. The antioxidants present in these food items reduce the adverse effects of both oxygenated and nitrogenous free radicals that are produced in the normal physiological functioning of the organism. But fruits and vegetables are not the only sources of antioxidants in the diet. Other foods like chocolate, coffee, tea, wine, beer and fresh herbs and apices $[5,6]$ also have an important contribution in antioxidant levels.

For years, multiples studies conducted predominantly in middle-aged populations have showed associations between antioxidant substances and all cause or cardiovascular disease mortality. Evidence that mortality may be associated with vitamin C, vitamin E, carotenoids and selenium intake or status has been obtained in several previous studies [7-11].

Given the diversity in the sources of antioxidants and the interaction that exists between the different nutrients of different food items that constitute our diet, is important to use an indicator that reflects adequately the daily exposure to antioxidants and that takes into account the synergic effect of all the antioxidants substances present in the food [12]. Dietary total antioxidant capacity measures the overall antioxidant capacity of diet and has been shown to be an indicator of diet quality $[13,14]$. Similarly to individual antioxidants, total dietary antioxidant capacity has also been inversely associated to stroke [13,15], cancer [16] and heart failure [17].

The antioxidant capacity of the diet determines chronic inflammatory states [18,19]. Given the relationship between the inflammation and some chronic diseases and death, the aim of the present study was to assess the association between the dietary total antioxidant capacity, the dietary intake of different antioxidant vitamins and mortality in a Mediterranean population at high cardiovascular risk. 


\section{MATERIALS AND METHODS}

\section{Study design}

This study was conducted within the frame of the PREDIMED study (PREvención con DIeta MEDiterránea): a randomized, multicenter, parallel group, single-blinded dietary intervention trial conducted in Spain with the aim to analyze the effect of the Mediterranean Diet (MeDiet) on the prevention of major cardiovascular disease. Participants were randomly assigned to three groups: MeDiet supplemented with extra virgin olive oil (MeDiet + EVOO), MeDiet with nuts (MeDiet + nuts), and the control group [20]. The main results of the trial on the primary end point have been published elsewhere [21].

The PREDIMED study included 7447 participants, men aged between 55 and 80 years and women between 60 and 80 years who were at high cardiovascular risk. Subjects, who had no cardiovascular disease at enrolment, met at least one of the following criteria: presence of type 2 Diabetes Mellitus (T2DM) or the presence of three or more cardiovascular risk factors (current smoking, hypertension, dyslipemia, overweight or obesity, or family history of premature CVD.

The present analysis was conducted as an observational prospective cohort study considering baseline data before the individuals were randomized to the intervention group. The protocol was approved by the institutional review boards of each recruitment center and all participants provided a written informed consent prior to their inclusion in the study

For this analysis, we excluded subjects without follow-up ( $\mathrm{n}=201)$, and with values of total energy intake outside of predefined limits ( $<800$ or $>4000 \mathrm{kcal} / \mathrm{d}$ in men and: $<500$ or $>3500 \mathrm{kcal} / \mathrm{d}$ in women) $(\mathrm{n}=231)$ [22]. Overall, 7,015 subjects were analysed in this study.

\section{Antioxidant capacity intake and dietary assessment}

Trained dieticians used a 137-item food frequency questionnaire (FFQ) to assess dietary habits by faceto-face interviews. This FFQ was repeatedly administered at baseline and each year during follow-up. The FFQ has been previously validated in a sample of participants of the PREDIMED study [23,24]. Energy (kcal/d) and nutrient intake (g/d) were calculated as frequency multiplied by nutrient concentration in a specified portion size, where frequencies were measured in nine categories for each food item. Nutrient data bank was updated using the latest available information included in food composition tables for Spain [25]. Alcohol intake was also ascertained through the use of this questionnaire.

Dietary total antioxidant capacity was estimated using published databases that provided the antioxidant capacity measured in foods by assays FRAP (ferric reducing antioxidant power) [6]. 
All-cause mortality was determined by review of the End Point adjudication Committee whose members were unaware of the interventions assignments or any dietary exposure. Information on all-cause mortality was updated yearly. The sources of information were initially obtained from the continuous contact with participants and their families that we had during the trial, contact with family physicians, the yearly comprehensive review of all medical records and by yearly consultation of the National Death Index. The analyses included cases confirmed between October 1st, 2003, and December 1st, 2010.

\section{Co-variates assessment}

At baseline socio-demographic information, medical history and use of medication and lifestyle habits were collected via specific questionnaires. Trained personnel carried out anthropometric and blood pressure measurements. Height and weight were measured wearing light clothes, barefoot, using a wallmounted stadiometer and calibrated scales. BMI was estimated as weight $(\mathrm{kg})$ divided by the height $\left(\mathrm{m}^{2}\right)$ squared. Physical activity was assessed using a validated Spanish version of the Minnesota leisure-time physical activity questionnaire [26].

\section{Statistical analysis}

Baseline characteristics of the population were summarized according to quintiles of dietary total antioxidant capacity. Follow up time was calculated from the date of recruitment to the date of either death or end of follow-up (the date of the last visit or the last recorded clinical event of participants still alive). To assess the risk of all-cause mortality by quintiles of antioxidant intake and quintiles of dietary antioxidant capacity different Cox regression models were used to estimate multivariable-adjusted hazard ratios (HR) and their 95\% confidence intervals.

We used Cox regression models with time-dependent exposures with update diet and covariates. For dietary measures, we used the cumulative average of food intakes from baseline to the censoring events.

To ascertain the association between the quintiles of dietary total antioxidant capacity and total mortality, a first model was adjusted for recruitment center, intervention group, age (years, continuous), sex, education (low, middle, high), marital status (married, other), BMI (underweight, normal, overweight and obese), smoking habit (never, past, current), leisure-time physical activity (METs-min/d score, continuous), alcohol intake (g/d, continuous), total energy intake (kcal/d, continuous), and self-reported history of cancer, arterial hypertension, history of dyslipidemia and cardiovascular disease (yes, no). A second model was additionally adjusted for energy-adjusted intake of saturated fatty acids (g/d), polyunsaturated fatty acids (g/d), monounsaturated fatty acids (g/d) and glycemix index. Tests of linear trend across increasing quintiles of dietary total antioxidant capacity were conducted by assigning the medians to each quintiles and these variables were treated as continuous in the multivariable models.

Moreover, to assess the presence of interactions between the dietary total antioxidant capacity and different variables such as intervention group, sex, obesity or smoking at baseline, product terms were built and included in the multivariable models. 
To estimate multivariable-adjusted HR and their 95\% CI for all-cause mortality according quintiles of each antioxidant intake the model was adjusted for the same variables used to adjust the second model in the dietary antioxidant capacity index analysis.

Statistical analyses were performed using SPSS software package for Windows version 19.0 (SPSS Inc., Chicago, IL, USA) and Stata 12.0 (StataCorp, College Station, TX, USA). The significance level was set at $\mathrm{p}<0.05$. 


\section{RESULTS}

The subjects in the highest quintile of dietary total antioxidant capacity were more likely to be younger, ex-smokers, physically more active, with secondary or university level of education and with higher alcohol intake. In contrast, subjects in the highest quintile of adherence to the antioxidant capacity index were less likely to have a history of hypertension. The antioxidant capacity index was also directly associated to alcohol and red wine consumption, fruit and cereal intake (table 1).

During follow-up (median 4.3 years), 319 deaths were reported. Table 2 shows the association between the quintiles of antioxidant capacity index and mortality risk. There was an inverse association with total mortality (multivariable HR: 0.85; 95\% CI: 0.60-1.20) and with mortality from cardiovascular disease (multivariable HR: 0.79; 95\% CI: 0.43-1.43), for participants in the highest quintile of antioxidant capacity index compared with participants in the lowest quintile, although these associations were not significant. Neither was significant the association for antioxidant capacity index and death from cancer. Additional adjustment for several dietary factors such as fatty acids and glycemic index did not change the reported results.

After repeating the analyses including several product terms in the multivariable models, only a significant interaction was found for dietary total antioxidant capacity and smoking at baseline for cancer mortality (p for interaction=0.046). Thus, the analysis for this outcome was re-analysed after stratifying the sample by smoking status (never/former vs. current smokers). No significant association between dietary total antioxidant capacity and cancer mortality was found among never and former smokers. However, an important reduction (around 80\%) in the risk of cancer death was reported for those participants who were current smokers and belonged to the third and fourth quintiles of dietary total antioxidant capacity as compared to those within the first category (reference). HR and 95\% CI were 0.18 (0.04-0.83) and 0.23 (0.07-0.79) respectively.

In the analysis of the effect of several single antioxidants and mortality (table 3), no significant association was found between vitamin intake and mortality risk. Although the estimates did not reach statistical significance, other antioxidants such as vitamin $C, \beta$-cryptoxanthin, phytosterols or selenium were inversely associated to the risk of death from any cause with reductions of around 20 to $30 \%$. HR and 95\% CI for extreme quintiles of vitamin C intake were: 0.78 (0.51-1.18); HR and 95\% CI for extreme quintiles of $\beta$ - cryptoxanthin intake were: 0.70 (0.48-1.02); HR and 95\% CI for extreme quintiles of phytosterols intake were: 0.73 (0.47-1.11); and HR and 95\% CI for extreme quintiles of selenium intake were: $0.74(0.49-1.10)$. 


\section{DISCUSSION}

In this large prospective study in a population of older subjects with cardiovascular risk factors, we found no association between dietary total antioxidant capacity of the diet and mortality. Only subjects in the fourth quintile of dietary total antioxidant capacity had a lower risk of all-cause mortality when comparing to subjects belonging to the first quintile. These differences disappeared when additionally the results were adjusted for fatty acids and glycemic index intake. Also subjects in the fourth quintile had a $32 \%$ lower risk of cancer mortality.

The results did not change after carrying out several sensitivity analyses (data not shown), excluding the deaths occurring in the first two years of follow-up or participants with less than two years of follow-up, including only participants aged with less 60,65 or 70 years at baseline or including only deaths occurring $<60$ or $<70$ years.

The beneficial effect that antioxidants have on health is well known. However, the action of individual antioxidants can not be entirely determined due to the complex synergistic interactions present among these substances. Total antioxidant capacity has been recognised as a new tool to investigate the relationship between dietary antioxidant intake and mortality/diseases, providing the anti-oxidant potential of the total diet rather than that of single nutrients [12]. The antioxidant capacity of the diet is consider a good indicator of plasma antioxidant status [27-29] and also an indicator of nutritional quality [14].

Several cohort studies have investigated the protective effect of dietary total antioxidant capacity on the risk of some diseases. However, we haven't found any study that related dietary total antioxidant capacity with mortality in elderly population. In the EPIC cohort, with a large number of subjects form different European countries and aged between 35 and 70 years, results showed that a high dietary intake of antioxidant capacity was associated with a reduced risk of gastric cancer [16]. A case-control study conducted in an Italian population found a consistent inverse relation between this index and colorectal cancer [30].

Regarding to cardiovascular disease, the results by Rautiainen et al in the Swedish Mammography Cohort, showed that the total capacity antioxidant of diet was associated with lower risk myocardial infarction [31] and heart failure [17]. In this cohort of Swedish women researches also found an inverse association between total capacity antioxidant of diet and haemorrhagic stroke but not with cerebral infarction [13]. On the contrary, Del Rio et al in the EPIC cohort suggested that a diet rich in total capacity antioxidant of diet reduce the risk of cerebral infarction but not the risk haemorrhagic stroke [15].

In the Rotterdam Study, a prospective cohort that included 5,395 participants aged 55 years and older, dietary total antioxidant capacity of the diet did not seem to predict the risk of major neurologic diseases [32]. Other cohort studies have shown an inverse association between dietary total antioxidant capacity, diabetes [33] and metabolic syndrome [34]. 
When the different dietary antioxidants were analysed separately, results showed that subjects in the highest quintile had a lower mortality risk than those belonging to the lowest intake for most of the antioxidants substances, but these differences were not significant. This finding is in concordance with the results of other studies also conducted in elderly people and where the evidence of the association between mortality and antioxidant vitamin intake is scanty $[8,35,36]$. However, in a previous analysis conducted also in the frame of the PREDIMED study [37] results showed an inverse relationship between polyphenol intake and mortality.

More evidence exists on the beneficial effect that the intake of fruits and vegetables with high antioxidant power has in the prevention of certain chronic diseases and in all-cause mortality $[1,2,38]$.

Though the epidemiological evidence shows that the consumption of food items rich in antioxidants is related to a lower risk of mortality and incidence of chronic diseases there is no clear evidence that the supplementation with high doses of antioxidant vitamins is effective in the reduction of the risk of this type of pathologies. On the other hand it has been suggested that the beneficial effect of dietary antioxidants is higher in subjects having some risk factors at baseline [39]. In our population the possibility of oxidative stress is high though this is an elderly population at high cardiovascular risk. Otherwise, the population studied in this analysis belongs to a dietary intervention trial in which three groups of intervention are established: a Mediterranean diet supplemented with olive oil group, a Mediterranean diet supplemented with mixed nuts group and a control group without dietetic supplementation who received advice to follow a low fat diet.

Nevertheless, and as a possible explanation of our findings it is probable that dietary antioxidants levels in this population were adequate from the beginning of the study though participants from the PREDIMED are diabetics or have at least three risk factors of cardiovascular disease. So, these conditions preclude the adoption of dietetic changes including the increase in the antioxidant intake.

The increase interest for the antioxidants substances has risen, also the development of methods to estimate the antioxidant value of the diet. Given the synergistic interactions between the different components of foods and the different mechanisms of action of each one it is more adequate to use a combination of them to evaluate with more accuracy the antioxidant capacity of the food [12]. The use of only this method to estimate the antioxidant capacity in our population could be a limitation of our study. In fact, other specific assays such as (e.g., ORAC or ABTS) could complete the dietary antioxidant capacity assessement [40].

Another fact to take into account is the influence that the dietary antioxidant capacity has on the total antioxidant capacity. In spite of the strong consistence existing of the direct role of dietary antioxidants in modulating the plasma antioxidant network it is necessary to consider the influence of multiple factors that can modify the bioavailability of the antioxidants and therefore their activity in vivo [41].

Moreover, non-differential measurement error in the assessment of dietary total antioxidant capacity may have biased our results. This bias is particularly important for this non-binary exposure [42]. The 
estimation of dietary total antioxidant capacity was derived through a food frequency questionnaire that included a limited number of food items. Thus, although the validity and reproducibility of the dietary questionnaire has been previously evaluated the antioxidant capacity of the diet could have been underestimated. Additionally, multiple factors including cultivation procedures, growing and harvesting conditions and food.

Some strengths of our study also deserve to be mentioned. They included its prospective design, its longterm follow up and the large simple size, thus it is not representative of general population. Also it is of great interest the use of repeated measurements for calculating the dietary intake which could be used to reduce measurement error due to intra-individual variation.

In conclusion, the results of this study in older people at high cardiovascular risk did not show any association between dietary antioxidant capacity and the risk of total mortality, cardiovascular mortality or cancer mortality. Further studies that combined this index with other indicators of antioxidant capacity are needed to analyse more accurately the relationship between the adherence to a diet rich in antioxidants and the occurrence of disease or death.

\section{Acknowledgments}

The authors want to thank the participants of the study for their collaboration and the PREDIMED personnel for their excellent assistance with all aspects of the trial.

This study was funded by the Spanish Ministry of Health (ISCIII), CIBEROBN, PI1001407, G03/140, RD06/0045 and the Autonomous Government of Catalonia, and Caixa Tarragona (10-1343). The Fundación Patrimonio Comunal Olivarero and Hojiblanca SA (Málaga, Spain), California Walnut Commission (Sacramento, CA), Borges SA (Reus, Spain) and Morella Nuts SA (Reus, Spain) donated the olive oil, walnuts, almonds and hazelnuts, respectively. None of the funding sources played a role in the design, collection, analysis, interpretation or publication of the data. AG is supported by a FPU fellowship from the Spanish Government

Conflict of interest. The authors declare that they have no conflict of interest. 


\section{REFERENCES}

1. Leenders M, Sluijs I, Ros MM, Boshuizen HC, Siersema PD, Ferrari P, Weikert C, Tjønneland A, Olsen A, Boutron-Ruault MC, Clavel-Chapelon F, Nailler L, Teucher B, Li K, Boeing H, Bergmann MM, Trichopoulou A, Lagiou P, Trichopoulos D, Palli D, Pala V, Panico S, Tumino R, Sacerdote C, Peeters PH, van Gils CH, Lund E, Engeset D, Redondo ML, Agudo A, Sánchez MJ, Navarro C, Ardanaz E, Sonestedt E, Ericson U, Nilsson LM, Khaw KT, Wareham NJ, Key TJ, Crowe FL, Romieu I, Gunter MJ, Gallo V, Overvad K, Riboli E, Bueno-de-Mesquita HB (2013) Fruit and vegetable consumption and mortality: European prospective investigation into cancer and nutrition. Am J Epidemiol 178:590-602.

2. Agudo A, Cabrera L, Amiano P, Ardanaz E, Barricarte A, Berenguer T, Chirlaque MD, Dorronsoro M, Jakszyn P, Larrañaga N, Martínez C, Navarro C, Quirós JR, Sánchez MJ, Tormo MJ, González CA (2007) Fruit and vegetable intakes, dietary antioxidant nutrients, and total mortality in Spanish adults: findings from the Spanish cohort of the European Prospective Investigation into Cancer and Nutrition (EPIC-Spain). Am J Clin Nutr 85:1634-1642.

3. Dauchet L, Amouyel P, Hercberg S, Dallongeville J (2006) Fruit and vegetable consumption and risk of coronary heart disease: a meta-analysis of cohort studies. J Nutr 136:2588-2593.

4. He FJ, Nowson CA, MacGregor GA (2006) Fruit and vegetable consumption and stroke: meta-analysis of cohort studies. Lancet 367:320-326.

5. Saura-Calixto F, Goñi I (2006) Antioxidant capacity of the Spanish Mediterranean diet. Food Chemistry 94:442-447.

6. Pellegrini N, Serafini M, Colombi B, Del Rio S, Salvatore S, Bianchi M, Brighenti F (2003) Total antioxidant capacity of plant foods, beverages and oils consumed in Italy assessed by three different in vitro assays. J Nutr 133:2812-2819.

7. Serafini M, Del Rio D (2004) Understanding the association between dietary antioxidants, redox status and disease: is the Total Antioxidant Capacity the right tool? Redox Rep 9:145-152.

8. Rautiainen S, Larsson S, Virtamo J, Wolk A (2012) Total antioxidant capacity of diet and risk of stroke: a population-based prospective cohort of women. Stroke 43:335-340.

9. Puchau B, Zulet MA, de Echávarri AG, Hermsdorff HH, Martínez JA (2009) Dietary total antioxidant capacity: a novel indicator of diet quality in healthy young adults. J Am CollNutr 28:648-656.

10. Bates CJ, Hamer M, Mishra GD (2011) Redox-modulatory vitamins and minerals that prospectively predict mortality in older British people: the National Diet and Nutrition Survey of people aged 65 years and over. Br J Nutr 105:123-132.

11. Fletcher AE, Breeze E, Shetty PS (2003) Antioxidant vitamins and mortality in older persons: findings from the nutrition add-on study to the Medical Research Council Trial of Assessment and Management of Older People in the Community. Am J Clin Nutr 78:999-1010.

12. Buijsse B, Feskens EJ, Schlettwein-Gsell D, Ferry M, Kok FJ, Kromhout D, de Groot LC (2005) Plasma carotene and alpha-tocopherol in relation to $10-\mathrm{y}$ all-cause and cause-specific mortality in European elderly: the Survey in Europe on Nutrition and the Elderly, a Concerted Action (SENECA). Am J ClinNutr 82:879-886.

13. Ray AL, Semba RD, Walston J, Ferrucci L, Cappola AR, Ricks MO, Xue QL, Fried LP (2006) Low serum selenium and total carotenoids predict mortality among older women living in the community: the women's health and aging studies. J Nutr 136:172-176.

14. Khaw KT, Bingham S, Welch A, Luben R, Wareham N, Oakes S, Day N (2001) Relation between plasma ascorbic acid and mortality in men and women in EPIC-Norfolk prospective study: a prospective population study. European Prospective Investigation into Cancer and Nutrition. Lancet 357:657-663. 
15. Del Rio D, Agnoli C, Pellegrini N, Krogh V, Brighenti F, Mazzeo T, Masala G, Bendinelli B, Berrino F, Sieri S, Tumino R, Rollo PC, Gallo V, Sacerdote C, Mattiello A, Chiodini P, Panico S (2011) Total antioxidant capacity of the diet is associated with lower risk of ischemic stroke in a large Italian cohort. J Nutr 141:118-123.

16. Serafini M, Jakszyn P, Luján-Barroso L, Agudo A, Bas Bueno-de-Mesquita H, van Duijnhoven FJ, Jenab M, Navarro C, Palli D, Boeing H, Wallström P, Regnér S, Numans ME, Carneiro F, BoutronRuault MC, Clavel-Chapelon F, Morois S, Grioni S, Panico S, Tumino R, Sacerdote C, Ramon Quirós J, Molina-Montes E, Huerta Castaño JM, Barricarte A, Amiano P, Khaw KT, Wareham N, Allen NE, Key TJ, Jeurnink SM, Peeters PH, Bamia C, Valanou E, Trichopoulou A, Kaaks R, Lukanova A, Bergmann MM, Lindkvist B, Stenling R, Johansson I, Dahm CC, Overvad K, Jensen M, Olsen A, Tjonneland A, Lund E, Rinaldi S, Michaud D, Mouw T, Riboli E, González CA (2012) Dietary total antioxidant capacity and gastric cancer risk in the European prospective investigation into cancer and nutrition study. Int J Cancer 131: E544-554.

17. Rautiainen S1, Levitan EB, Mittleman MA, Wolk A (2013) Total antioxidant capacity of diet and risk of heart failure: a population-based prospective cohort of women. Am J Med 126:494-500.

18. Brighenti F, Valtueña S, Pellegrini N, Ardigò D, Del Rio D, Salvatore S, Piatti P, Serafini M, Zavaroni I (2005) Total antioxidant capacity of the diet is inversely and independently related to plasma concentration of high-sensitivity C-reactive protein in adult Italian subjects. Br J Nutr 93:619-625.

19. Valtueña S, Pellegrini N, Franzini L, Bianchi MA, Ardido D, del Rio D, Piatti P, Scazzina F, Zavaroni I, Brighenti F (2008) Food selection based on total antioxidant capacity can modif. antioxidant intake, systemic inflammation, and liver function without altering markers of oxidative stress. Am J ClinNutr 87:1290-1297.

20. Martínez-González MÁ, Corella D, Salas-Salvadó J, Ros E, Covas MI, Fiol M, Wärnberg J, Arós F, Ruíz-Gutiérrez V, Lamuela-Raventós RM, Lapetra J, Muñoz MÁ, Martínez JA, Sáez G, Serra-Majem L, Pintó X, Mitjavila MT, Tur JA, Portillo MP, Estruch R; PREDIMED Study Investigators (2012) Cohort profile: design and methods of the PREDIMED study. Int J Epidemiol 41:377-385.

21. Estruch R, Ros E, Salas-Salvadó J, Covas MI, Corella D, Arós F, Gómez-Gracia E, Ruiz-Gutiérrez V, Fiol M, Lapetra J, Lamuela-Raventos RM, Serra-Majem L, Pintó X, Basora J, Muñoz MA, Sorlí JV, Martínez JA, Martínez-González MA; PREDIMED Study Investigators (2013) Primary prevention of cardiovascular disease with a Mediterranean diet. N Engl J Med 368:1279-1290.

22. Willet W (1998) Issues in analysis and presentation of dietary data. In: Willet W (ed) Nutritional Epidemiology, 2nd edn. Oxford University Press, New York, pp 321-346.

23. Fernández-Ballart JD, Piñol JL, Zazpe I, Corella D, Carrasco P, Toledo E, Perez-Bauer M, MartínezGonzález MA, Salas-Salvadó J, Martín-Moreno JM (2010) Relative validity of a semi-quantitative foodfrequency questionnaire in an elderly Mediterranean population of Spain. Br J Nutr 103:1808-1816.

24. de la Fuente-Arrillaga C, Vázquez Z, Bes-Rastrollo M, Sampson L, Martínez-González MA (2010) Reproducibility of an FFQ validated in Spain. Public Health Nutr 13:1364-1372.

25. Mataix L (2003) Tabla de composición de Alimentos Españoles. Granada, Spain

26. Elosua R, Marrugat J, Molina P, Pons S, Pujol E (1994) Validation of the Minnesota leisure time physicalactivityquestionnaire in Spanishmen. The MARATHOM Investigators. Am J Epidemiol 139:1197-1209.

27. Wang Y, Yang M, Lee SG, Davis CG, Koo SI, Chun OK (2012) Dietary total antioxidant capacity is associated with diet and plasma antioxidant status in healthy young adults. J Acad Nutr Diet 112:16261635.

28. Yang M, Chung SJ, Floegel A, Song WO, Koo SI, Chun OK (2013) Dietary antioxidant capacity is associated with improved serum antioxidant status and decreased serum C-reactive protein and plasma homocysteine concentrations. Eur J Nutr 52:1901-1911. 
29.Valtueña S, Del Rio D, Pellegrini N, Ardigò D, Franzini L, Salvatore S, Piatti PM, Riso P, Zavaroni I, Brighenti F (2007) The total antioxidant capacity of the diet is an independent predictor of plasma betacarotene. Eur J Clin Nutr 61:69-76.

30. La Vecchia C, Decarli A, Serafini M, Parpinel M, Bellocco R, Galeone C, Bosetti C, Zucchetto A, Polesel J, Lagiou P, Negri E, Rossi M (2013) Dietary total antioxidant capacity and colorectal cancer: a large case-control study in Italy. Int J Cancer 133:1447-1451.

31. Rautiainen S1, Levitan EB, Orsini N, Åkesson A, Morgenstern R, Mittleman MA, Wolk A (2012) Total antioxidant capacity from diet and risk of myocardial infarction: a prospective cohort of women. Am J Med 125:974-980.

32. Devore EE, Feskens E, Ikram MA, den Heijer T, Vernooij M, van der Lijn F, Hofman A, Niessen WJ,Breteler MM (2013). Total antioxidant capacity of the diet and major neurologic outcomes in older adults. Neurology 80:904-910.

33. Okubo H, Syddall HE, Phillips DI, Sayer AA, Dennison EM, Cooper C, Robinson SM; Hertfordshire Cohort Study Group (2014) Dietary total antioxidant capacity is related to glucose tolerance in older people: the Hertfordshire Cohort Study. Nutr Metab Cardiovasc Dis 24:301-308.

34. Bahadoran Z, Golzarand M, Mirmiran P, Shiva N, Azizi F (2012) Dietary total antioxidant capacity and the occurrence of metabolic syndrome and its components after a 3-year follow-up in adults: Tehran Lipid and Glucose Study. Nutr Metab (Lond) 9:70.

35. Genkinger JM, Platz EA, Hoffman SC, Comstock GW, Helzlsouer KJ (2004) Fruit, vegetable, and antioxidant intake and all-cause, cancer, and cardiovascular disease mortality in a community-dwelling population in Washington County, Maryland.

Am J Epidemiol 160:1223-1233.

36. Roswall N, Olsen A, Christensen J, Hansen L, Dragsted LO, Overvad K, Tjønneland A (2012) Micronutrient intake in relation to all-cause mortality in a prospective Danish cohort. Food Nutr Res 56.

37. Tresserra-Rimbau A, Rimm EB, Medina-Remón A, Martínez-González MA, López-Sabater MC, Covas MI, Corella D, Salas-Salvadó J, Gómez-Gracia E, Lapetra J, Arós F, Fiol M, Ros E, Serra-Majem L, Pintó X, Muñoz MA, Gea A, Ruiz-Gutiérrez V, Estruch R, Lamuela-Raventós RM; PREDIMED Study Investigators (2014) Polyphenol intake and mortality risk: a re-analysis of the PREDIMED trial. BMC Med 12:77.

38. Hjartåker A1, Knudsen MD, Tretli S, Weiderpass E (2014) Consumption of berries, fruits and vegetables and mortality among 10,000 Norwegian men followed for four decades. Eur J Nutr Aug 3

39. Serafini M, Miglio C, Peluso I, Petrosino T (2011) Modulation of plasma non enzimatic antioxidant capacity (NEAC) by plant foods: the role of polyphenols. Curr Top Med Chem 11:1821-1846.

40. Takashima M, Horie M, Shichiri M, Hagihara Y, Yoshida Y, Niki E (2012) Assessment of antioxidant capacity for scavenging free radicals in vitro: a rational basis and practical application. Free Radic Biol Med 52(7):1242-1252.

41. Frankel EN, Meyer AS (2000) Review. The problems of using one-dimensional methods to evaluate multifunctional food and biological antioxidants. J Sci Food Agric 80:1925-1941.

42. Wacholder S, Hartge P, Lubin JH, Dosemeci M (1995) Non-differential misclassification and bias towards the null: a clarification. Occup Environ Med 52(8):557-558 